\title{
O OLHAR DOS ADOLESCENTES SOBRE A VIOLÊNCIA E O LAZER NOS TERRITÓRIOS PELAS LENTES DO PHOTOVOICE
}

\section{THE LOOK OF ADOLESCENTS OVER VIOLENCE AND THE LEISURE IN THE TERRITORIES BY THE LENSES OF PHOTOVOICE}

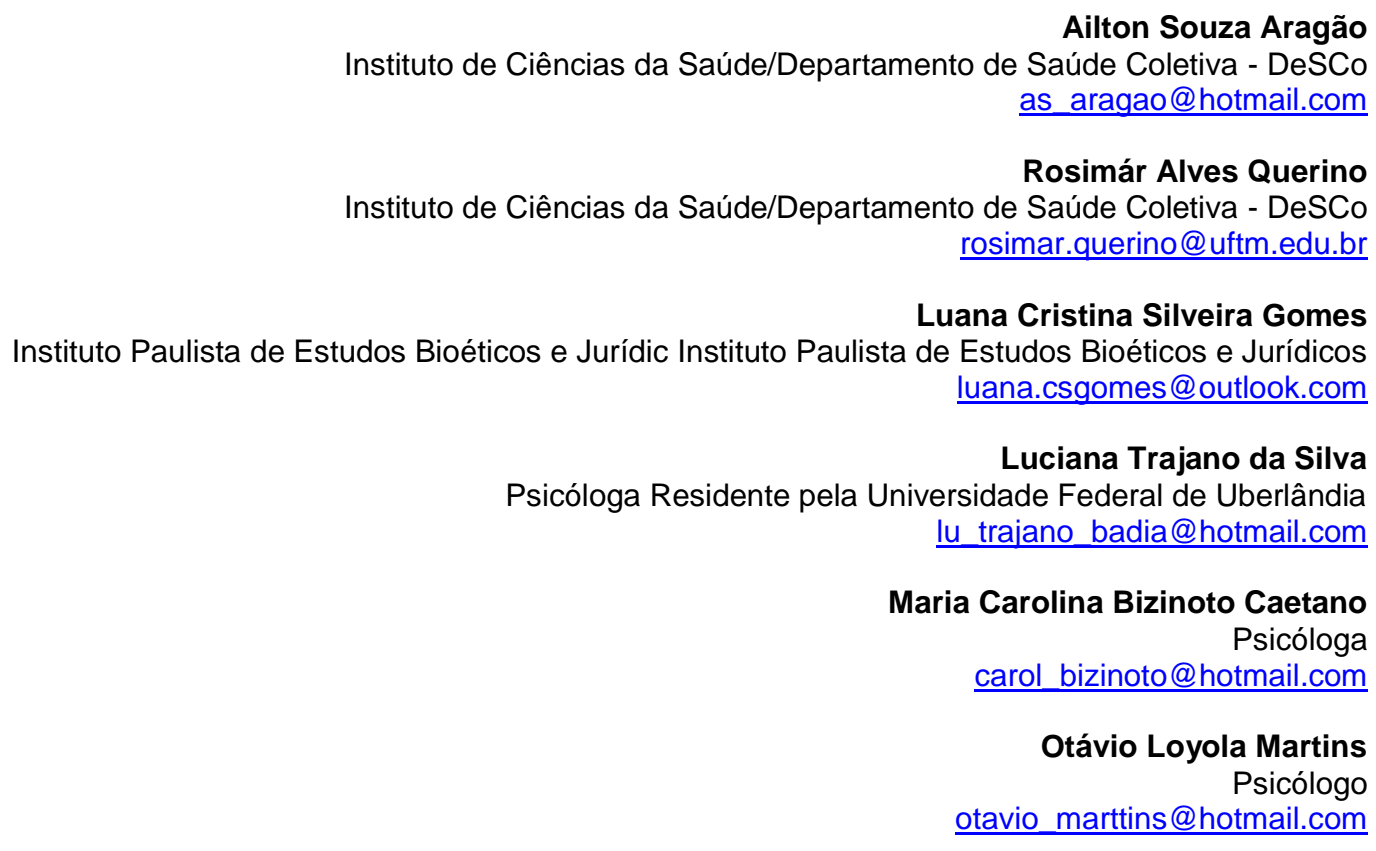

\author{
Ana Angelina Amatângelo Oliveira \\ Graduanda em Psicologia pela Universidade Federal do Triângulo Mineiro \\ anamatangelo@hotmail.com \\ Ana Carolina Graner Araújo Oliveira \\ Graduanda em Psicologia pela Universidade Federal do Triângulo Mineiro \\ anacarolina-16@hotmail.com \\ Maria Lopes Santos \\ Graduanda em Psicologia pela Universidade Federal do Triângulo Mineiro \\ lopezinha2011@hotmail.com \\ Fabiano Henrique Oliveira Sabino \\ Graduando em Enfermagem pela Universidade Federal do Triângulo Mineiro \\ fabianooliveira163@gmail.com
}

\begin{abstract}
RESUMO
A violência noticiada sob a forma de espetáculo favorece a produção de julgamentos apressados que ocultam seus determinantes sociais e engendram intervenções que reforçam a lógica ostensiva e a reclusão dos segmentos populacionais historicamente vulnerados, dentre estes, os adolescentes. Considerando-os como sujeitos de direitos e produtores de uma linguagem própria, as fotografias permitem desvelar os discursos que criminalizam a pobreza. A pesquisa objetivou analisar a apreensão de 77 adolescentes frequentadores de um programa de fortalecimento de vínculos e convivência comunitária denominado ProJovem Adolescente, em funcionamento nos Centros de Referência de Assistência Social (CRAS), sobre seus territórios durante o ano de 2015 e 2016. Adotou-se o "photovoice", seguido da audiogravação, transcrição das narrativas, categorização e análise de conteúdo temática. As imagens e as narrativas categorizadas retratam a violência e os limites para o lazer nos
\end{abstract}

Recebido em: 09/07/2018

Aceito para publicação em: 14/02/2019 
O olhar dos adolescentes sobre a violência e o lazer nos territórios pelas lentes do photovoice
Ailton Souza Aragão

Rosimár Alves Querino

Luana Cristina Silveira Gomes

Luciana Trajano da Silva

Maria Carolina Bizinoto Caetano

Otávio Loyola Martins

Ana Angelina Amatângelo Oliveira

Ana Carolina Graner Araújo Oliveira

Maria Lopes Santos

Fabiano Henrique Oliveira Sabino

territórios: as pichações nas praças, as depredações de equipamentos de uso público; a presença de terrenos baldios e, fundamentalmente, o medo promovido pelo tráfico de drogas. As vulnerabilidades territoriais, como a ausência de equipamento públicos, evidenciam a violência estrutural que fragiliza e reduz a proteção aos riscos da violência, a qual colabora para a estigmatização das comunidades. A linguagem fotográfica e as narrativas estimulam o engajamento dos adolescentes e colabora para o empoderamento dos mesmos.

Palavras-chave: Adolescentes. Território. Violência.

\section{ABSTRACT}

The violence reported in the form of a spectacle favors the production of hurried judgments that conceal its social determinants and engender interventions that reinforce the ostensible logic and the reclusion of historically violated population segments, among which, the adolescents. Considering them as subjects of rights and producers of their own language, the photographs allow us to unveil the discourses that criminalize the poverty. The research aimed to analyze the apprehension of 77 adolescents attending of a program of strengthening of community ties and coexistence called ProJovem Adolescent, in operation in the Centers of Reference of Social Assistance, about their territories during the year 2015 and 2016. The " photovoice" was adopted, followed by audio-recording, transcription of narratives, categorization and analysis of thematic content. The categorized images and narratives depict violence and limits to leisure in territories: grafitti on squares, depredations of public use equipment; the presence of vacant lots and, fundamentally, the fear promoted by drug dealing. Territorial vulnerabilities, such as the absence of public equipment, evidence the structural violence that weakens and reduces the protection of the risks of violence, which contributes to the stigmatization of communities. The photographic language and the narratives stimulate the engagement of adolescents and contribute to their empowerment.
\end{abstract}

Keywords: Adolescents. Territory. Violence.

\title{
INTRODUÇÃO
}

Tem sido consenso na literatura que o adolescer deve ser considerado como processo social, compreendido em suas contradições. Desse modo, o adolescer não está restrito ao caráter biológico ou psicológico, pois ambos os processos de desenvolvimento estão imbricados em contextos territoriais e também históricos. Logo, há a necessidade de compreensão contextualizada do processo de vida.

Certo é que o subsídio da discussão da experiência dos adolescentes é a afirmação de sua condição de cidadãos, presente no arcabouço jurídico e político desde a década de $1990 \mathrm{com}$ a promulgação do Estatuto da Criança e do Adolescente (ECA), em 13 de julho de 1990 (BRASIL, 2002). Esta perspectiva é completamente diversa daquela que vigorou ao longo do século XX, expressa de modo lapidar no Código de Menores de 1927.

Em sua afirmação histórico-jurídica o ECA opera uma transformação nos direitos das crianças e adolescentes ao situá-los no rol dos intensos debates jurídicos e políticos que ensejam os Direitos Humanos. Em território nacional, a Constituição Federal de 1988 emerge das lutas por cidadania, manifestos no Artigo 6으, denominados direitos sociais. No Artigo 227 prenuncia o que será o ECA ao enunciar que o zelo pelas crianças e adolescentes é de responsabilidade da família, da sociedade e do Estado, reconhecendo-os como sujeitos de direitos e dignos de prioridade absoluta (CARVALHO, 2008; BRASIL, 2017).

Os estudos de Carvalho e Buss (2008) se apoiando em Dahlgren e Whitehead (1991) dentre outros apontam os determinantes sociais de saúde como um modelo explicativo do processo saúde-doença que agrega vários elementos e acena para a necessidade de se trabalhar com as dimensões micro e macroestruturais do processo de vida. Assim, esses autores definiram diversos planos de determinação 
O olhar dos adolescentes sobre a violência

e o lazer nos territórios pelas lentes do photovoice
Ailton Souza Aragão

Rosimár Alves Querino

Luana Cristina Silveira Gomes

Luciana Trajano da Silva

Maria Carolina Bizinoto Caetano

Otávio Loyola Martins

Ana Angelina Amatângelo Oliveira

Ana Carolina Graner Araújo Oliveira

Maria Lopes Santos

Fabiano Henrique Oliveira Sabino

do processo saúde-doença: idade, sexo e fatores hereditários; estilos de vida dos indivíduos; redes sociais e comunitárias e condições socioeconômicas, culturais e ambientais gerais.

Desse modo, os impactos dessa análise na elaboração de políticas e na organização de serviços são inovadores, pois remetem ao enfrentamento das "causas das causas" que impactam nos processos saúde-enfermidade - esta compreendida em sua experiência subjetiva. Compreensão que evidencia a superação dos modelos estritamente biológicos, focados na doença em sua manifestação física. Essa assertiva aponta para a necessidade de irmos para além do sistema de atenção à saúde pois reconhece-se que as intervenções nas condições socioeconômicas, ambientais e culturais são fundantes de novas ações, aqui, as interdisciplinares e intersetoriais. "[...] E, sobretudo, em políticas de desenvolvimento, voltadas para a distribuição mais equânime dos recursos socialmente produzidos, subordinando a economia ao bem-estar social" (CARVALHO; BUSS, 2008, p. 160).

O debate avança na direção da efetivação de análises do processo saúde-doença com a adoção do modelo de determinantes sociais de saúde encontra no conceito de vulnerabilidades denso aporte teórico-medotológico. Na definição de Ayres et al. (2003) podemos apreender as vulnerabilidades em três dimensões: vulnerabilidade individual, vulnerabilidade social e vulnerabilidade programática.

Por seu turno, Dachs, Bambas e Casas (2005), corroboram tal interpretação ao discutir os impactos da iniquidade em saúde, ou seja, não temos somente indivíduos vulnerabilizados, temos segmentos sociais e comunidades inteiras vivenciando processos de vulnerabilização que resultam de macrodeterminantes e, portanto, de processos políticos mais amplos. Assim, o enfrentamento das vulnerabilidades demanda, por exemplo, o envolvimento comunitário e a participação política nos processos decisórios e na formulação de políticas públicas capazes de contribuir com a autonomia dos sujeitos diante dos tipos de violência que se manifestam nos territórios e impactam, sobretudo, crianças e adolescentes.

Por violência compreendemos, como disposto por Krug et al. (2002):

[...] o uso de força física ou poder, em ameaça ou na prática, contra si próprio, outra pessoa ou contra um grupo ou comunidade que resulte ou possa resultar em sofrimento, morte, dano psicológico, desenvolvimento prejudicado ou privação (KRUG et al., 2002, p. 5).

De um lado, o conceito reporta o poder que, na prática da violência, é assimétrico, desigual entre os envolvidos na relação de violência. Assim, desde uma desigualdade na força física, manifesta na violência doméstica; à violência econômica, como as sanções comerciais de um país sobre outro, a violência possui uma intencionalidade, que pode ou não produzir danos imediatos. Logo, ainda que a violência não produza ferimentos ou a morte imediata em suas vítimas, ela poderá produzir efeitos no desenvolvimento integral de crianças e adolescentes, por exemplo, pela privação de alimentos ou de medicamentos (KRUG, et al., 2002; DAHLBERG, KRUG, 2007).

Nessa direção, nos territórios em que agudiza-se as vulnerabilidades, pela ausência de políticas públicas, por exemplo, evidenciam-se as naturezas e as manifestações das violências sobre segmentos populacionais frágeis fisicamente, como crianças e adolescentes (KRUG, et al., 2002; DAHLBERG, KRUG, 2007). Em território nacional, segundo o Atlas da Violência - 2017, os indicadores de homicídios revelam um traço comum. Suas vítimas são, em geral, jovens entre 15 e 29 anos; negros, desempregados, com acesso precarizado à escola e residências de áreas urbanas degradadas (CERQUEIRA et al., 2017).

Os estudos demonstram, ainda, que as políticas públicas de enfrentamento das violências devem incidir sobre os determinantes sociais. Nisso reside a promoção da saúde, enquanto atuação estratégica intersetorial de base territorial e como crítica aos modelos emergenciais e fragmentados (VILLASANTE, 2002; MIOTO, 2002; NJAINE et al., 2006 ARAGÃO et al., 2013).

Compreender o adolescer como processo nos auxilia na apreensão crítica da concepção etária e biológica desse segmento demográfico e na crítica ao modelo assistencialista de política social e suas implicações históricas. Uma vez que esse modelo se limita ações emergenciais, episódicas e 
O olhar dos adolescentes sobre a violência e o lazer nos territórios pelas lentes do photovoice
Ailton Souza Aragão

Rosimár Alves Querino Luana Cristina Silveira Gomes Luciana Trajano da Silva Maria Carolina Bizinoto Caetano Otávio Loyola Martins Ana Angelina Amatângelo Oliveira Ana Carolina Graner Araújo Oliveira Maria Lopes Santos

Fabiano Henrique Oliveira Sabino

segmentadas que, calcada, sobretudo na lógica filantrópica, tende a reduzir os direitos sociais a favores privados (na efetividade dos direitos sociais de crianças e adolescentes). Buscar superá-las é construir novos saberes que promovam o debate e a proposição de ações que incluam, por exemplo, a territorialidade da produção da saúde do adolescente em meio às violências (AYRES et al., 2003; SILVA JÚNIOR, 2006; BATISTELLA, 2007; FERNANDEZ; MENDES, 2007).

Nisso reside a relevância de apreender as perspectivas dos adolescentes sobre os territórios por meio de metodologias participativas, como o photovoice, e que venham a produzir um diagnóstico sobre as violências vivenciadas e como estas impactam no processo de adolescer nesses territórios.

\section{METODOLOGIA}

Trata-se de estudo de caso de tipo exploratório desenvolvido com a combinação de métodos quantitativos e qualitativos. Participaram da pesquisa 77 adolescentes de oito Coletivos ProJovem, entre os anos de 2014 e 2015.

Para a construção dos dados fora adotada a técnica photovoice, que destaca a relevância da produção iconográfica dos participantes. O método se caracteriza por um processo pelo qual os adolescentes são dotados de um papel ativo e capazes de captar as nuances que se alteram nos territórios vividos sob a forma de imagens. Estas, serão selecionadas pelos mesmos e convertidas em narrativas que buscam situar o fotógrafo-sujeito no território à luz de suas experiências, de temores, desejos, expectativas, realizações, projetos (WANG, BURRIS, 1997; DELGADO, 2015).

O photovoice é uma estratégia metodológica que, a partir de fotografias produzidas pelos próprios sujeitos, fomenta a participação e conscientização dos mesmos acerca das dificuldades individuais e comunitárias (WANG, BURRIS, 1997). Segundo Caroline Wang e Mary Ann Burris (1994) e Melvin Delgado (2015) sua inspiração, dentre outras, reside nos escritos de Paulo Freire, haja vista o potencial educativo da produção de fotografia participativa como método de pesquisa, ou uma "educação para a consciência crítica". Uma vez que o processo que acompanha essa estratégia valoriza os sujeitos, considerando-os dotados de um papel ativo e catalizador de mudança na utilização combinada da foto com as histórias contadas.

No tocante aos adolescentes dos territórios dos Centros de Referência em Assistência Social (CRAS), Hernandez, Shabazian e McGrath (2014) e Delgado (2015), referindo-se a outros autores, sintetizam que a entrega de uma câmera fotográfica para os mesmos se traduz num processo participativo que Ihes permite analisar criticamente a comunidade em que vivem, fomentam a exposição de narrativas diferentes das que comumente se obtêm com uma entrevista, estimulam a escuta daqueles que geralmente não o são, dada a condição de marginalidade ou de estigmatização.

Nessa mesma direção Robert Strack et al. (2015) destacam que o photovoive, além de permitir construir uma documentação dos pontos fortes/fracos ou questões particulares das comunidades sob a forma de imagens, essa captação estimula a exposição oral e/ou redigida dos participantes e o posterior debate coletivo sobre as realidades vividas e compartilhadas. O resultado pode ser, sobretudo, encorajar a comunidade à ação participativa por meio do debate com os operadores políticos, por exemplo.

Esse é um método bastante utilizado com cidadãos marginalizados e estigmatizados, a fim de oferecer oportunidades de observação e discussão crítica de situações referentes à realidade vivenciada por tal público, além de favorecer o empoderamento de ações transformadoras (HERNANDEZ, SHABAZIAN, MCGRATH, 2014).

Por sua vez, Schell et al. (2009) concordam ao afirmarem que o photovoice possibilita que os indivíduos não apenas percebam os impasses locais, mas sim os analisem e discutam a respeito, com o intuito de pensar em alternativas de transformação. Essa constatação é significativa pois de acordo com Wang et al. (2004), a consciência crítica é promovida a partir do englobamento de questões de natureza histórica, social e política. 
O olhar dos adolescentes sobre a violência e o lazer nos territórios pelas lentes do photovoice
Ailton Souza Aragão

Rosimár Alves Querino

Luana Cristina Silveira Gomes

Luciana Trajano da Silva

Maria Carolina Bizinoto Caetano

Otávio Loyola Martins

Ana Angelina Amatângelo Oliveira

Ana Carolina Graner Araújo Oliveira

Maria Lopes Santos

Fabiano Henrique Oliveira Sabino

Em sua operacionalidade, antes de deixarmos o CRAS, os pesquisadores conduziram uma breve explicação sobre o funcionamento das câmeras digitais e seus recursos. Uma surpresa para muitos, haja vista a presença dos smartphones com alguns deles. Coletaram-se alguns dados como idade e sexo para facilitar a identificação dos fotógrafos e sua produção, sobretudo na etapa da transcrição.

Um segundo momento, foi a exposição da pergunta "de acordo com o seu ponto de vista, o que produz saúde e adoecimento no seu bairro?". As muitas respostas foram sendo construídas com fornecimento de câmeras digitais em que os adolescentes guiaram os pesquisadores pelos muitos bairros/comunidades em que estão instalados os CRAS na cidade pesquisada, em caminhadas de cerca de duas horas. Lembrando que a maioria residia no entorno do CRAS. Foram realizadas oito oficinas de photovoice nos oito coletivos de ProJovem durante o ano de 2014 e 2015.

$\mathrm{Na}$ etapa seguinte, os adolescentes selecionaram três imagens do total de sua produção, estas foram exibidas num telão com o objetivo de lhe captar a interpretação da imagem, à luz de suas experiências nos territórios, com seus dilemas e potenciais. Ao mesmo tempo, à interpretação do fotógrafo-sujeito, fora se somando a outras experiências e interpretações similares dos demais adolescentes, cujas aproximações permitiram ampliar a compreensão da realidade/cenário retratado. Os relatos foram audiogravados e as imagens foram identificadas como forma de lhes aderir ao/aos depoimentos, e posteriormente, as narrativas foram transcritas. Para o presente, nos valemos do conjunto de fotografias do acervo produzidas pelos/pelas 77 adolescentes, como forma de ampliar a compreensão dos territórios dos CRAS e em função das categorias temáticas que emergiram das narrativas sobre as fotografias selecionadas.

A análise na modalidade de conteúdo temática primou pela construção de categorias temáticas, em que as imagens produzidas foram motivadoras de uma avaliação dos territórios. Segundo Bardin (1979) o processo do photovoice se insere no rol de:

[...] técnicas de análise de comunicação visando obter, por procedimentos sistemáticos e objetivos de descrição do conteúdo das mensagens, indicadores (quantitativos ou não) que permitam a inferência de conhecimentos relativos às condições de produção/recepção destas mensagens (BARDIN, 1979, p.42)

A análise de conteúdo temática pressupõe (a) contato exaustivo com o material; (b) apreensão das particularidades do material gerado pela pesquisa original, identificação e recorte temático dos dados considerando as questões norteadoras do estudo; (c) organização do material, considerando critérios de exaustividade, representatividade, homogeneidade e pertinência (constituição do corpus); (d) determinação de ideias centrais dos dados, organizadas em gavetas de investigação; (e) análise e exploração do material, visando alcançar os núcleos temáticos e, na sequência, as temáticas; e (f) elaboração da síntese interpretativa a partir das categorias temáticas (MINAYO, 2010).

Buscando a identificação das formas da violência presente nos territórios vividos pelos adolescentes e como estas influem em sua saúde, emergiram duas categorias temáticas, a saber: Drogas: entre os traficantes e os usuários, os adolescentes e Sentidos de lazer, lugares da sociabilidade nos territórios.

Em atenção à Resolução 466/2012, do Conselho Nacional de Saúde, nomeamos os participantes com uma sigla (Ad. 1, Ad.2, etc.), e os nomes dos CRAS que frequentavam fora nomeado com um numeral romano (CRAS I, CRAS II, etc.), como forma de manter o anonimato dos adolescentes e dos/das trabalhadores/as.

\section{RESULTADOS E DISCUSSÃO}

\section{Drogas: entre os traficantes e os usuários, os adolescentes}

O tema das drogas é recorrente entre os adolescentes frequentadores dos Coletivos. Suas imagens e narrativas revelam um cenário atravessado pela presença do tráfico, dos usuários e, praticamente, de ausência do Estado, entendida como força repressiva e ostensiva. Contudo, há elementos que tornam o cenário ainda mais complexo, como a violência familiar e social, bem como a adoção dos espaços 
O olhar dos adolescentes sobre a violência

e o lazer nos territórios pelas lentes do photovoice
Ailton Souza Aragão

Rosimár Alves Querino Luana Cristina Silveira Gomes

Luciana Trajano da Silva

Maria Carolina Bizinoto Caetano

Otávio Loyola Martins

Ana Angelina Amatângelo Oliveira

Ana Carolina Graner Araújo Oliveira

Maria Lopes Santos

Fabiano Henrique Oliveira Sabino

públicos pelos usuários de drogas para consumo.

A problemática das drogas se mostra multifacetada quanto aos seus determinantes (CARVALHO; BUSS, 2008). Porém, as iniciativas do poder público têm se reduzido à higiene social: dissuasão dos usuários das áreas centrais das cidades por meio da adoção de forças policiais de caráter ostensivo, ou ainda, pela internação (voluntária, involuntária ou compulsória), em geral, em comunidades terapêuticas privadas.

O tema adquire maior relevância quando adolescentes dos territórios e em condição especial de desenvolvimento são colocados diuturnamente em contato com traficantes e usuários e passam a ser assediados para o consumo e/ou para a venda de drogas, realidade comum nos grandes centros, como constatou Nancy Cardia et al. (2012), mas também verificável pelos próprios adolescentes:

\footnotetext{
[...] Fica fumando no banco [da praça], daí começa, daí já entra chutando a bola. Ad. 1 CRAS I

[...] e essa esquina aqui é os pontos dos 'noiados', sempre andam com uma arma na mão. Ad. 2 CRAS I

E é fácil comprar droga [...] é só você chegar 'ai dá um' e eles já te entregam e você leva embora. Ad. 3 CRAS IV

Cara eu morava perto de uma [boca de fumo] eu não vou saber? Eu já vi pedra no chão... assim ó [aponta para o chão]. Ad. 4 CRAS IV
}

A exposição constante dos adolescentes ao tráfico e ao uso de drogas se converte em violação de Direito Fundamental, logo, uma forma de violência, na medida em que têm Direito ao Convívio Social e Comunitário (BRASIL, ECA, 1990; CARDIA et al., 2012). Porém, os relatos indicam espaços "sitiados" no interior dos bairros, cujos acessos são controlados pelos traficantes ou pelos usuários de drogas. Verifica-se que o território vivido dos adolescentes guarda significados intrínsecos à lógica do mercado das drogas: espaços proibidos, códigos de conduta, logística e acesso facilitado às drogas.

Figura 1: Inscrição em mureta de quadra poliesportiva no bairro onde se localiza o CRAS III. 2015

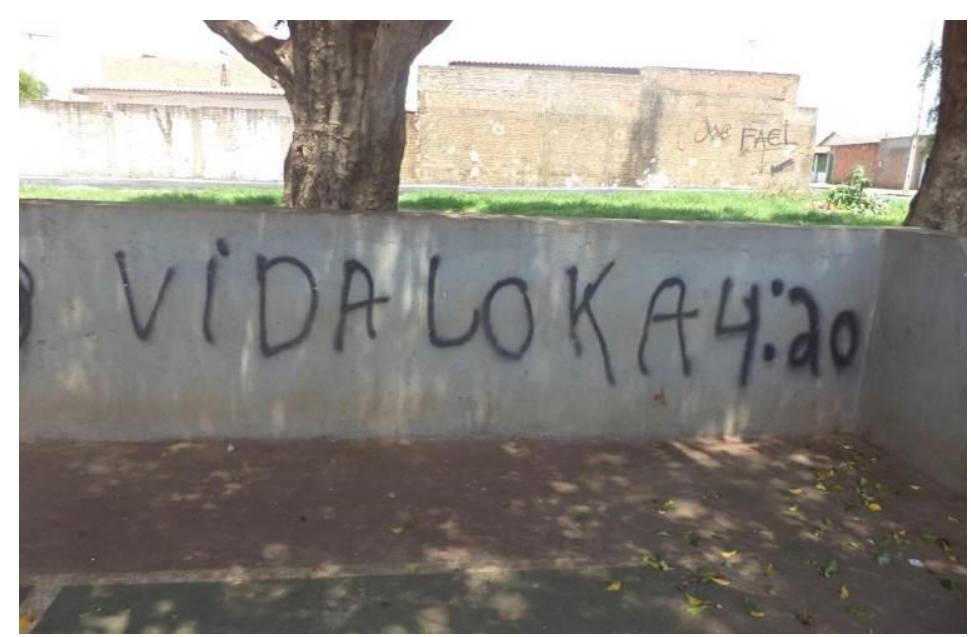

Autor: Fotógrafa adolescente. 14 anos. Fonte: Arquivo de imagens dos pesquisadores.

A situação de vulnerabilidade econômica do grupo familiar, maus tratos físicos ou psicológicos no ambiente doméstico, abandono da escola associados ao imperativo de socialização pelo consumo, necessidade de autoafirmação são alguns dos elementos que facultam o ingresso do adolescente no tráfico de drogas, satisfazendo todas as demandas de imediato (PINSKY; BESSA, 2004). Uma das estratégias pode ser a entrega de mercadorias furtadas por usuários de drogas e que podem ser 
O olhar dos adolescentes sobre a violência

e o lazer nos territórios pelas lentes do photovoice
Ailton Souza Aragão

Rosimár Alves Querino Luana Cristina Silveira Gomes

Luciana Trajano da Silva

Maria Carolina Bizinoto Caetano

Otávio Loyola Martins

Ana Angelina Amatângelo Oliveira

Ana Carolina Graner Araújo Oliveira

Maria Lopes Santos

Fabiano Henrique Oliveira Sabino

vendidas pelos adolescentes.

Uma vez eu passei e o cara ofereceu um celular pra vender, roubado, queria que eu vendesse por 8 reais e falei 'ocê tá doido'? Ad. 5. CRAS I

Esse cenário tem desafiado os agentes sociais institucionais que atuam na promoção dos direitos dos adolescentes: a compreensão do fenômeno ultrapassa a mera atuação sob os seus efeitos, como retirar os usuários dos locais de circulação da comunidade, em geral, e dos adolescentes, em particular. Uma vez que o fenômeno do consumo de drogas impacta nos comportamentos de todos os moradores de um determinado território.

Do lado da minha casa tem um cara que bate na 'muié', aí ela perdeu o neném dela.

Ad. 6 CRAS IV

O povo aqui é muito violento. Ad. 4 CRAS IV

A violência associada ao tráfico de drogas produz relações de desrespeito aos direitos dos adolescentes e de outros sujeitos vulneráveis, como mencionado acima. Mas o tráfico de drogas demonstra seu real poder contra aqueles que não honram seus compromissos, situação que demonstra seu modus operandi "pedagógico" aos olhos da comunidade e dos adolescentes.

Por causa ele tava devendo droga, aí ele não pagou e fizeram isso com ele [...] Deram um tiro na boca dele, na garganta e outro na bunda, e depois tacaram fogo nele. Ad. 7 CRAS I

Considerando o conceito de violência adotado (KRUG et al., 2002), o Adolescente 8 reconhece os sinais das muitas demonstrações de força do tráfico. Mas não só: há a força física do homem contra uma mulher ou o constante assédio dos usuários sobre os adolescentes que podem ser compreendidos como formas de violência, tais como a interpessoal doméstica e a comunitária (KRUG et al., 2002). Todas são manifestações de violência que denunciam, cada uma a seu modo, a não efetividade do acesso às políticas públicas de proteção social de base territorial. Logo, incidirão negativamente no desenvolvimento integral dos adolescentes (ECA, 1990).

Figura 2: Inscrição em muro de equipamento privado de esportes no bairro. CRAS II. 2015

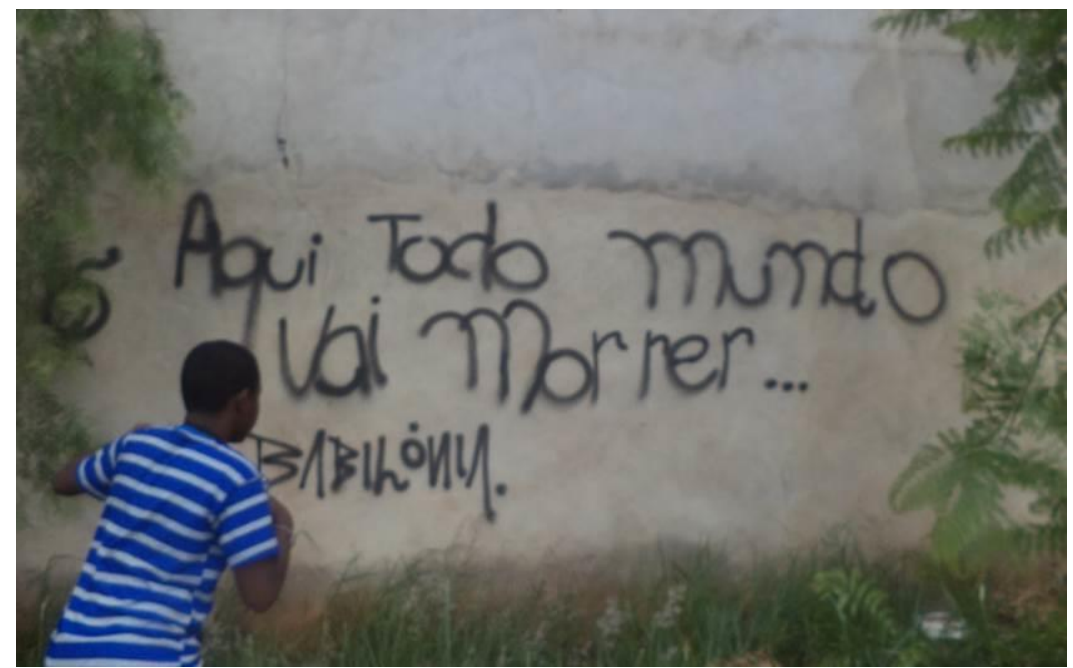

Autor: Fotógrafo adolescente. 14 anos. Fonte: Arquivo de imagens dos pesquisadores.

Acerca da Imagem, o adolescente assim revela seu temor:

Você pode mexer com a Babilônia, você toma um tiro. Ad. 8 CRAS II 
O olhar dos adolescentes sobre a violência

e o lazer nos territórios pelas lentes do photovoice
Ailton Souza Aragão

Rosimár Alves Querino Luana Cristina Silveira Gomes

Luciana Trajano da Silva

Maria Carolina Bizinoto Caetano

Otávio Loyola Martins

Ana Angelina Amatângelo Oliveira

Ana Carolina Graner Araújo Oliveira

Maria Lopes Santos

Fabiano Henrique Oliveira Sabino

A violência do tráfico de drogas é atravessada por outras violências de caráter social, logo, históricas, como o abandono de espaços urbanos, dentre praças e habitações, que serão ocupados por usuários de drogas. Com isso, acrescemos que há outras naturezas das violências que, como a social, estão há gerações fomentando outras formas de violências nos territórios, como a psicológica, a interpessoal, dentre outras.

Figura 3: Construção para fins habitacionais abandonada e usada como ponto de venda e uso de drogas no bairro. CRAS II. 2015

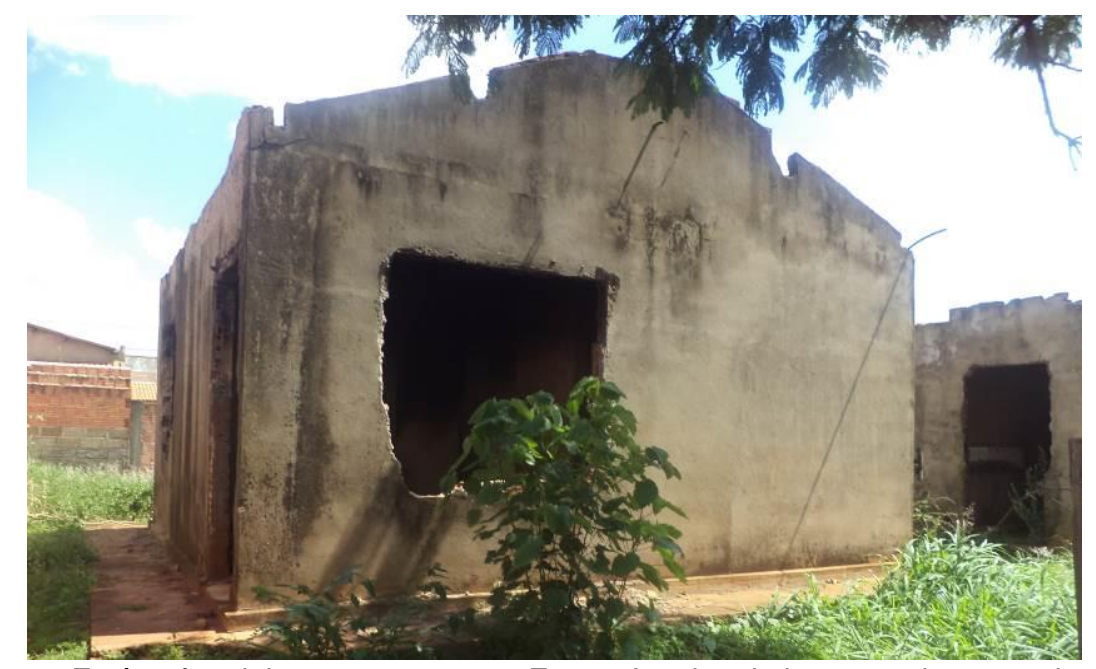

Autor: Fotógrafo adolescente. 15 anos. Fonte: Arquivo de imagens dos pesquisadores.

As violências manifestas são, dialeticamente, produzidas-produtoras-reprodutoras de vulnerabilidades, às quais engendram novas violências. Ou seja, a sobrevivência diária no território depende do ajustamento dos adolescentes ao modus operandi das muitas formas de violência, como forma de protegerem-se. São nesses cenários de vulnerabilidades históricas que estão os indicadores de homicídios entre adolescentes e jovens no Brasil, entre 12 e 19 anos, residentes em territórios de alta vulnerabilidade, negros, do sexo masculino (KRUG et al., 2002; MINAYO, 2009; BRASIL, UNICEF, 2010).

\section{Sentidos de lazer, lugares da sociabilidade nos territórios}

"[...] No lugar conhecemos o mundo pelo que ele já é, mas, também, pelo que ainda não é. Desta forma, o futuro, mais que o passado, torna-se nossa âncora (SANTOS, 1996, p.34)

As imagens e as narrativas dos espaços de lazer nos territórios demonstram que essa categoria pode ser subdividida entre "espaços de lazer" e "atividades de lazer". Essa estratégia permite uma compreensão dos usos que os adolescentes fazem dos espaços comunitários/públicos ou privados em relação aos tipos de atividades preferidas.

$\mathrm{Na}$ acepção de Rodrigues (2015), referindo-se aos textos de Milton Santos, os "lugares", enquanto atribuição de sentido em sua singularidade e de construção de identidade pelos sujeitos, são processos mediados pela dialética da cidade capitalista e global. Assim, dentre os lugares preferidos dos adolescentes dos oito Coletivos estão as igrejas; as sorveterias; as quadras; as praças e os clubes particulares; campo de futebol; shopping; padaria; Centro Municipal de Educação Avançada; fazenda e casa. 
O olhar dos adolescentes sobre a violência e o lazer nos territórios pelas lentes do photovoice
Ailton Souza Aragão

Rosimár Alves Querino Luana Cristina Silveira Gomes

Luciana Trajano da Silva

Maria Carolina Bizinoto Caetano

Otávio Loyola Martins

Ana Angelina Amatângelo Oliveira

Ana Carolina Graner Araújo Oliveira

Maria Lopes Santos

Fabiano Henrique Oliveira Sabino

No tocante às praças, Silva e Carvalho (2017) ao estudar uma na cidade de Londrina, enfatizam seu potencial como equipamento de promoção da saúde e qualidade de vida, reforçando, assim, o disposto na Política Nacional de Promoção da Saúde (BRASIL, 2010), que preconiza, dentre outros aspectos, a importância de se "valorizar e otimizar o uso dos espaços públicos de convivência e de produção de saúde para o desenvolvimento das ações de promoção da saúde (p.17). Logo, a promoção da saúde extrapola o cotidiano dos moradores que residem nos arredores da praça, pois sua manutenção (ou não) impactará na vida cotidiana de todos os moradores, portanto, de sua saúde.

Dentre as atividades preferenciais dos adolescentes em seus territórios estão jogar futebol; soltar pipa, queimada, assistir jogos de futebol pela televisão e educação física, internet/redes sociais, brincar com cães nas ruas, festas na quadra.

É sabida a importância do lazer como meio para o desenvolvimento de crianças e adolescentes, pois além se configurar com um dos Direitos Fundamentais expressos no Estatuto da Criança e do Adolescente (BRASIL, 1990), sua efetividade, por meio de políticas públicas, promove o desenvolvimento biológico, psíquico e social da criança e do adolescente, portanto, da saúde considerada em sua integralidade, fazendo coro com o preconizado na Carta de Ottawa (BRASIL, 2010).

Figura 4: Praça utilizada pelos adolescentes do Coletivo do CRAS II para atividades recreativas. 2015

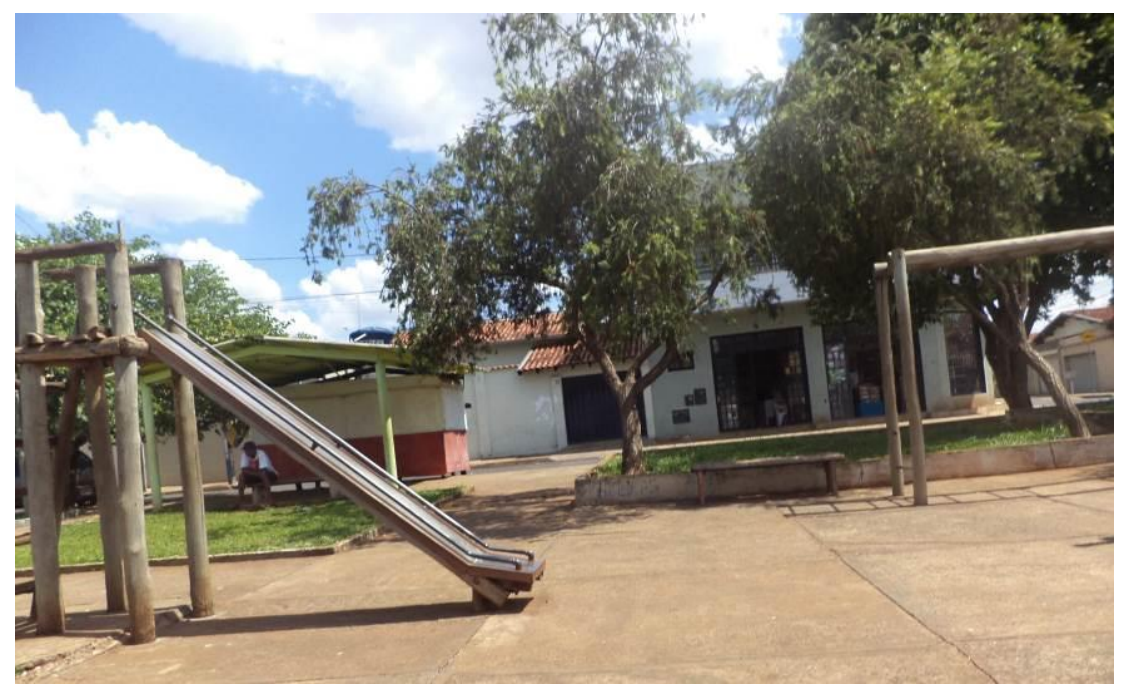

Autor: Fotógrafo adolescente. 14 anos. Fonte: Arquivo de imagens dos pesquisadores.

Assim, para além das formas de sociabilidade preconizadas pela sociedade de consumo aos adolescentes, em particular, como os shoppings, nos territórios se desdobra o processo de sociabilidade desses adolescentes por meio das atividades de lazer. Isso não significa dizer que os adolescentes não frequentem os shoppings da cidade, porém, como frequentadores do ProJovem, eram estimulados a conhecer e a se apropriarem dos espaços do bairro, fazendo do mesmo, um lugar (RODRIGUES, 2015). Nessa direção, o photovoice proporcionou o fortalecimento dessa premissa disposta nas diretrizes dos Serviços de Convivência e Fortalecimento de Vínculos (BRASIL, 2014).

As atividades expostas pelos adolescentes permitem as trocas simbólicas, o desenvolvimento de estratégias de negociação, a administração de conflitos, a apropriação do território, o desenvolvimento da tolerância bem como a abertura para novas relações e experiências no território vivido.

[...] vamos jogar bola, curtir, lugar fresco [na praça]. Ad. 8 CRAS III

Ahh, e lá [na praça] é bom pra jogar bola." Ad. 9 CRAS I 
O olhar dos adolescentes sobre a violência e o lazer nos territórios pelas lentes do photovoice
Ailton Souza Aragão

Rosimár Alves Querino Luana Cristina Silveira Gomes

Luciana Trajano da Silva

Maria Carolina Bizinoto Caetano

Otávio Loyola Martins

Ana Angelina Amatângelo Oliveira

Ana Carolina Graner Araújo Oliveira

Maria Lopes Santos

Fabiano Henrique Oliveira Sabino

[...] É uma quadra perto da minha casa. (...) gosto de jogar só futebol" [...] Com os amigos". Ad. 10 CRAS V

[...] Eu gosto de ir pra igreja. Participo do grupo de jovens. Ad. 11 CRAS III

Tem missa dia de quarta, domingo e sábado, que sábado eu faço catequese. Ad. 12 CRAS II

Tem dia que eu vou com a [Orientadora Social], pra jogar bola. Ad. 13 CRAS II

A praça, a quadra, a igreja e o campo de futebol são espaços de sociabilidade disponíveis em praticamente todos os territórios dos adolescentes e por eles são utilizados como forma de proteção contra os riscos presentes nos territórios de vulnerabilidade, mas também de descontração, de interação social, ou ainda de compartilhamento de significados.

Figura 5: Quadra poliesportiva utilizada pelos adolescentes de do Coletivos de ProJovem do CRAS III. 2015

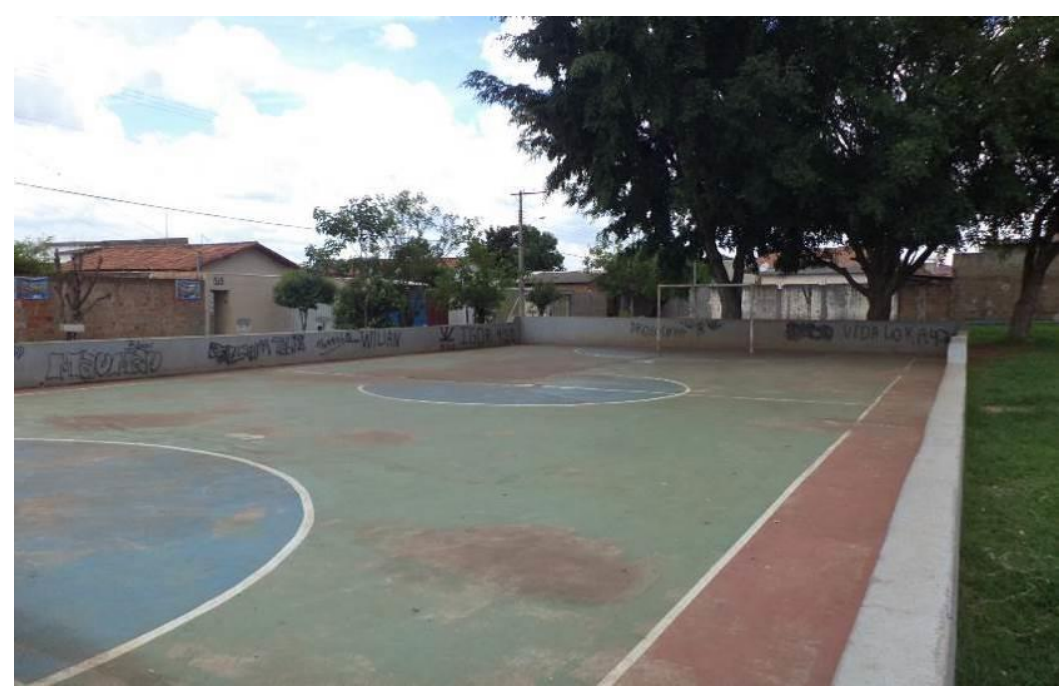

Autor: Fotógrafo adolescente. 15 anos. Fonte: Arquivo de imagens dos pesquisadores.

Como se observa, o equipamento construído - quadra - é o mesmo que fora fotografado por outro adolescente do mesmo Coletivo. No entanto, o mesmo lugar adquire significados diferentes que dependem das experiências dos adolescentes com o lugar, se de segurança, conforto, identidade solidariedade ou de ameaça, instabilidade, exposição ao risco, competição; de variáveis como sexo, etnia; se faz uso de drogas; distância da residência em relação ao lugar. Esses lugares, como a quadra, são, como salienta Milton Santos (2008) catalisadores de como os adolescentes, as pessoas, podem passar a perceber o mundo ao seu redor e por esse lugar, balizar suas experiências em outros níveis, paralelos, como extensão homóloga à casa, ou hierárquicos, numa relação com a cidade.

É a quadra que eu ia com meu pai. [...] o que eu não gosto é que antes de estar escrito como tá aí, pichada, ela era bonita, aí agora picharam e ela ficou feia. Ad. 12 CRAS $\mathrm{V}$

Esses significados aliam, como reforça Rodrigues (2015), as experiências cotidianas de cada adolescente a um movimento dialético das transformações pelas quais o equipamento - quadra - irá passar em sua historicidade e, concomitante, às suas próprias vidas. Seja, por exemplo, pela ausência de manutenção periódica que, como destaca Silva e Carvalho (2017), são efeitos das contradições das políticas urbanas neoliberais que pouco valorizam os espaços públicos de lazer nos bairros, pois privilegiam os espaços para o consumo em massa, como os shoppings sob a égide do capitalismo. Destarte, outras formas de uso/significado são atribuídas ao lugar, como para o consumo de drogas ou 
O olhar dos adolescentes sobre a violência e o lazer nos territórios pelas lentes do photovoice
Ailton Souza Aragão

Rosimár Alves Querino Luana Cristina Silveira Gomes

Luciana Trajano da Silva

Maria Carolina Bizinoto Caetano

Otávio Loyola Martins

Ana Angelina Amatângelo Oliveira

Ana Carolina Graner Araújo Oliveira

Maria Lopes Santos

Fabiano Henrique Oliveira Sabino

de depredação.

Isso posto, a identificação da quadra como lugar de usuários de drogas nos territórios próximos aos CRAS, como exposto na Imagem 1, revelará a pluralidade que os territórios oferecem para o lazer dos adolescentes, que ora expressam a resistência, a denúncia, o inconformismo, a saudade. As experiências plurais experimentadas pelos adolescentes em outros espaços remetem à produção de novos significados, que não obedecem à mera reprodução funcional de ajustamento.

O moço nem deixa entrar lá [no clube privado] tem vez. [...] Uai, se der [para pular a cerca] ninguém segura né? Ad. 11 CRAS III

Tem gente que vai lá [no clube privado] pra namorar, é isso? Ad. 13 CRAS III

[...] Ô na roça é bão porque você tem lugar pra fazer tudo. Ad. 14 CRAS III

[...] tem mais igreja que casa. Ad. 4 CRAS IV

[...] não, não tem nenhum lugar, só um [a sorveteria]. Ad. 3 CRAS IV

A entrada proibida num clube privado, a exaltação do espaço da zona rural, a insatisfação com grande número de igrejas e a existência de apenas uma sorveteria no bairro revela que os adolescentes dos territórios pesquisados requerem de mais espaços de sociabilidade e mesmo para o consumo. Mas não quaisquer espaços: estes devem ser atrativos, que combinem os aparelhos de um clube privado, inclusive com piscinas, com a amplitude de um espaço rural e a oferta de atividades prazerosas, como tomar sorvete e poder namorar em segurança.

Figura 6: Centro Municipal de Educação Avançada em bairro próximo ao CRAS III e ao CRAS VI. 2014

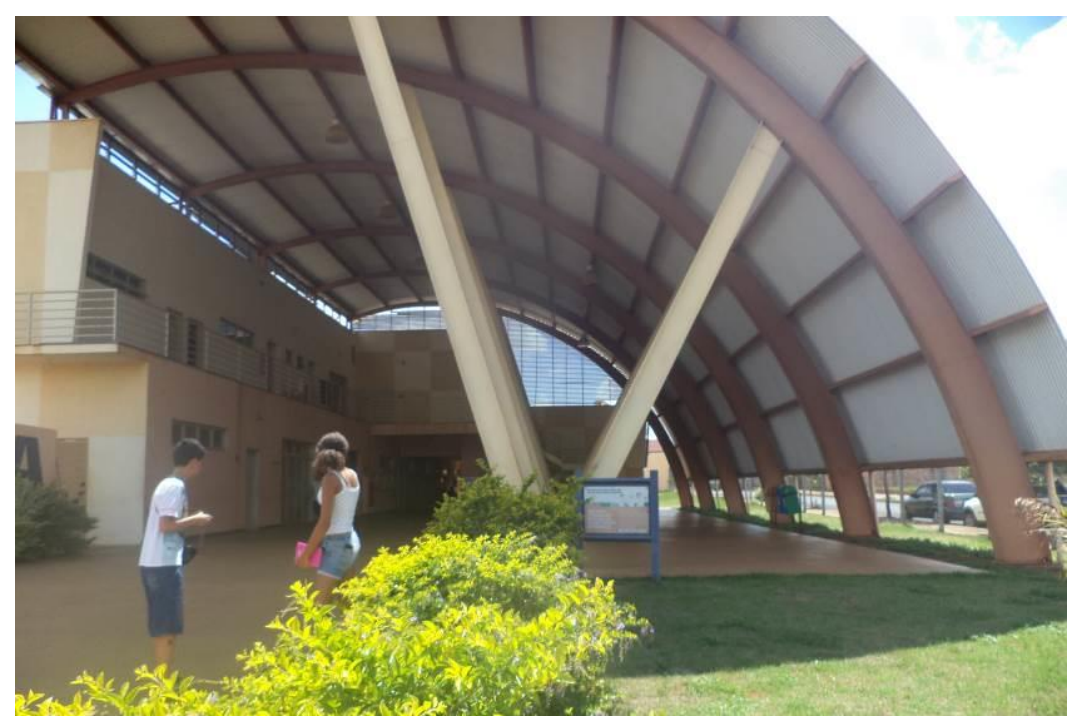

Autor: Fotógrafo adolescente. 15 anos. Fonte: Arquivo dos pesquisadores.

Aqui as praças e ruas, clubes, campos de futebol e demais equipamentos inevitavelmente se convertem nesses múltiplos espaços/lugares de sociabilidade dos adolescentes de ambos os sexos, de todas as idades. Logo, os espaços públicos permitem os encontros entre os adolescentes para práticas esportivas; de recreação, como soltar pipas, jogar queimada; ou de cultura, como participar de festas populares nas quadras dos bairros, ou seja, Ihes permite manifestar sua singularidade e pluralidade, simultaneamente. Ao passo que resistem, comunitariamente, ao abandono do lugar para o uso dos usuários de drogas.

Sob a ótica do lazer, são adolescentes do mesmo território e essa é uma categoria que os aglutina: pela experiência vivencial das ausências de espaços de lazer originários de políticas públicas 
O olhar dos adolescentes sobre a violência

e o lazer nos territórios pelas lentes do photovoice
Ailton Souza Aragão

Rosimár Alves Querino Luana Cristina Silveira Gomes

Luciana Trajano da Silva

Maria Carolina Bizinoto Caetano

Otávio Loyola Martins

Ana Angelina Amatângelo Oliveira

Ana Carolina Graner Araújo Oliveira

Maria Lopes Santos

Fabiano Henrique Oliveira Sabino

participativas; espaços que oportunizem e que congreguem as expectativas de um momento particular do desenvolvimento biopsicossocial dos mesmos. E que cujas buscas implicam, mesmo, na ruptura com o acesso privado ao lazer a qual pode ser interpretada como forma de resistir, individual ou coletivamente, à uma sociedade estruturalmente violenta que os coloca e os mantêm à margem da efetividade do direito ao lazer, por exemplo (ARAGÃO et al., 2013; MINAYO, 2009)

$O$ acesso a esses espaços de lazer pode gerar e fortalecer o sentimento de pertencimento, de reconhecimento e de identidade pessoal e social, de valorização do território, sem conduzir a monolitismos de natureza anatomofisiológica, políticas ou jurídicas, posto que essas identidades são dotadas de historicidade espaço-temporal, próprios da condição de ser do adolescente (STRACK, MAGILL, McDONAGH, 2004).

\section{CONCLUSÃO}

O tráfico e o uso de drogas se converteram em experiências cotidianas dos/das adolescentes, e para além do senso comum, têm ameaçado a segurança e a integridade psicossocial dos mesmos. Essa realidade nos territórios os obriga a construírem estratégias de enfrentamento, como se ausentarem dos espaços públicos de uso coletivo, com as praças e quadras esportivas. Estratégia que os impele a limitarem as formas de sociabilidade como forma de proteção às ameaças que o tráfico de drogas e os usuários passam a representar.

A efetivação do direito ao lazer nos territórios, como sinônimo de promoção da saúde, não se efetiva para todos os/as adolescentes. A busca por alternativas passa pelo questionamento das políticas públicas, como forma de expressão da vulnerabilidade, e da superação da lógica do lazer como resultante da capacidade de consumo dos mesmos e suas famílias.

Nessa direção, as fotografias e as narrativas dos adolescentes evidenciam que a presença do tráfico de drogas nos espaços destinados ao lazer limita a realização de atividades de promoção da saúde pelas equipes da Atenção Básica destinadas aos próprios adolescentes e à comunidade. Cenário que desafia a emersão da potencialidade política do próprio território.

O direito ao lazer; à dignidade, ao respeito e à liberdade; direito ao convívio social e comunitário, como dispostos no ECA, enfrenta limites estruturais para sua efetividade por meio de políticas públicas. $O$ subfinanciamento e a gestão fraudulenta dessas políticas reforçam as vulnerabilidades territoriais. Contudo, a efetividade desses direitos se revela nas estratégias criadas pelos próprios adolescentes a partir das possibilidades verificadas nos próprios territórios. Assim, de um lado não podemos considerar os adolescentes como meramente destinatários das políticas públicas locais ou mesmo federais, posto que, por meio do photovoice, diagnostica-se o potencial desse ciclo de vida no reconhecimento dos limites e das potencialidades presentes nos próprios territórios.

\section{REFERÊNCIAS}

ARAGÃO, A. S. et al. Abordagem dos casos de violência à criança pela enfermagem na atenção básica. Revista Latino-Americana de Enfermagem. Ribeirão Preto, SP. 2013. v.21, esp. p. 1-7. Disponível em <http://dx.doi.org/10.1590/S0104-11692013000700022>. Acesso em: 17 jan. 2018.

AYRES, J. R. C. M. et al. O conceito de vulnerabilidade e as práticas de saúde: novas perspectivas e desafios. In: CZERESNIA, D.; FREITAS, C. M. de (Org.). Promoção da saúde: conceitos, reflexões, tendências. Rio de Janeiro: FIOCRUZ, 2003. p. 117-139.

BARDIN, L. Análise de conteúdo. Lisboa: Edições 70, 1979.

BATISTELLA, C. Abordagens contemporâneas do conceito de saúde. In: FONSECA, A. F., CORBO, A. D. (Org.). O território e o processo saúde e doença. Rio de Janeiro: ESPJV, FIOCRUZ, 2007. p. 51-86. 
O olhar dos adolescentes sobre a violência

e o lazer nos territórios pelas lentes do photovoice
Ailton Souza Aragão

Rosimár Alves Querino Luana Cristina Silveira Gomes

Luciana Trajano da Silva

Maria Carolina Bizinoto Caetano

Otávio Loyola Martins

Ana Angelina Amatângelo Oliveira

Ana Carolina Graner Araújo Oliveira

Maria Lopes Santos

Fabiano Henrique Oliveira Sabino

BRASIL. Presidência da República. Estatuto da criança e do adolescente. Lei Federal n 8069 , de 13 de julho de 1990. Rio de Janeiro: Imprensa Oficial, 2002. Disponível em:

<http://www.planalto.gov.br/ccivil_03/leis//8069.htm>. Acesso em: 03 jun. 2018.

. Senado. Constituição Da República Federativa Do Brasil - Texto consolidado até a Emenda Constitucional no 97 de 04 de outubro de 2017. 2017. Disponível em

<http://www.senado.leg.br/atividade/const/con1988/con1988_04.10.2017/CON1988.pdf>. Acesso em: 14 dez. 2017.

. Secretaria de Vigilância em Saúde. Política Nacional de Promoção da Saúde, Brasília: Ministério da Saúde: 3.ed. 2010. Disponível em:

<http://bvsms.saude.gov.br/bvs/publicacoes/politica_nacional_promocao_saude_3ed.pdf>. Acesso em: 03 jun. 2018.

. Ministério do Desenvolvimento Social e Combate à Fome. Secretaria Nacional de Assistência Social. Conselho Nacional de Assistência Social. Resolução no 109, de 11 de novembro de 2009. Aprova a Tipificação Nacional de Serviços Socioassistenciais. Reimpressão 2014. Disponível em:

<http://www.mds.gov.br/webarquivos/publicacao/assistencia_social/Normativas/tipificacao.pdf>. Acesso em: 14 set. 2015.

. Ministério da Saúde. Secretaria de Atenção em Saúde. Departamento de Ações Programáticas Estratégicas. Diretrizes nacionais para a atenção integral à saúde de adolescentes e jovens na promoção, proteção e recuperação da saúde. Brasília: Ministério da Saúde, 2010. Disponível em:

<http://bvsms.saude.gov.br/bvs/publicacoes/diretrizes_nacionais_atencao_saude_adolescentes_joven s_promocao_saude.pdf>. Acesso em: 20 mar. 2013.

, UNICEF. Homicídios na adolescência no Brasil: IHA 2005/2007. Brasília, DF: Secretaria de Direitos Humanos, 2010.

CERQUEIRA, D. et al. (Org). Atlas da violência - 2017. Rio de Janeiro: Instituto de Pesquisa Econômica Aplicada, FBSP, 2017. Disponível em:

<http://www.ipea.gov.br/portal/images/170602_atlas_da_violencia_2017.pdf>. Acesso em: 03 jun. 2018.

CARDIA, N. et al. Pesquisa nacional, por amostragem domiciliar, sobre atitudes, normas culturais e valores em relação à violação de direitos humanos e violência: Um estudo em 11 capitais de estado. São Paulo: Núcleo de Estudos da Violência da Universidade de São Paulo, 2012.

CARVALHO, A. M.P. A luta por direitos e a afirmação das políticas sociais no brasil contemporâneo. Revista de Ciências Sociais. Fortaleza, CE. v.39, n.1, 2008. p. 16-26. Disponível em <http://www.rcs.ufc.br/edicoes/v39n1/rcs_v39n1a2.pdf>. Acesso em: 10 fev. 2018.

CARVALHO, A. I.; BUSS, P. M.. Determinantes sociais na saúde, na doença e na intervenção. In: GIOVANELLA, L. et al. (Org.). Políticas e sistemas de saúde no Brasil. Rio de Janeiro: FIOCRUZ, 2008. p. 141-166.

DACHS, J. N. W.; BAMBAS, A.; CASAS, J. A. Determinantes sociais e econômicos de desigualdades em saúde. In: MINAYO, M. C. S.; COIMBRA JÚNIOR, C. E. A. (Org.). Críticas e atuantes. Ciências humanas e sociais em saúde na América Latina. Rio de Janeiro: Fiocruz, 2005. p. 333-354.

DAHLBERG, L. L.; KRUG, E. G. Violência: um problema global de saúde pública. Ciência e Saúde Coletiva. Rio de Janeiro. 2006. v.11, supl. p.1163-1178. Disponível em:

$<$ http://www.scielo.br/scielo.php?script=sci_arttext\&pid=S1413-

$81232006000500007 \& \operatorname{lng}=\mathrm{en} \& \mathrm{nrm}=$ iso >. Acesso em: 24 de jun. 2017.

DAHLGREN, G., WHITEHEAD, M. Policies and strategies to promote social equity in health. Stockholm: Institute for Future Studies, 1991. Disponível em: 
O olhar dos adolescentes sobre a violência

e o lazer nos territórios pelas lentes do photovoice
Ailton Souza Aragão

Rosimár Alves Querino Luana Cristina Silveira Gomes

Luciana Trajano da Silva

Maria Carolina Bizinoto Caetano

Otávio Loyola Martins

Ana Angelina Amatângelo Oliveira

Ana Carolina Graner Araújo Oliveira

Maria Lopes Santos

Fabiano Henrique Oliveira Sabino

<https://core.ac.uk/download/pdf/6472456.pdf>. Acesso em: 23 jun. 2018.

https://doi.org/10.1590/S1413-81232006000500007

DELGADO, M. Urban youth and photovoice. New York: Oxford University, 2015.

FERNANDEZ, J. C. A.; MENDES, R. Gestão local e políticas públicas para a qualidade de vida. In: FERNANDEZ, J. C. A.; MENDES, R. (Org.). Promoção da saúde e gestão local. São Paulo: Aderaldo \& Rothschild, 2007. p.41-60.

KRUG, E. G. et al (Org.). Relatório mundial sobre violência e saúde. Genebra: OMS, 2002. Disponível em <http://www.opas.org.br/wp-content/uploads/2015/09/relatorio-mundial-violenciasaude.pdf >. Acesso em: 10 de mar. 2017.

HERNANDEZ, K.; SHABAZIAN, A. N.; MCGRATH, C. Photovoice as a pedagogical tool: examining the parallel learning processes of college students and preschool children through service learning. Creative Education. Los Angeles, USA. 2014. v.5, p.1947-1957. Disponível em: <http://dx.doi.org/10.4236/ce.2014.522219>. Acesso em: 10 mar. 2018. https://doi.org/10.4236/ce.2014.522219

MINAYO, M. C. S. Conceitos, teorias e tipologias de violência: a violência faz mal à saúde individual e coletiva. In: NJAINE, K.; ASSIS, S. G.; CONSTANTINO, P. (Org.). Impactos da violência na saúde. 2.ed. Rio de Janeiro: Fiocruz; EAD-ENSP, 2009. p.21-42. https://doi.org/10.7476/9788575415887.003

O desafio do conhecimento: pesquisa qualitativa em saúde. São Paulo: Hucitec, 2010.

MIOTO, R. C. T. O trabalho com redes como procedimento de intervenção profissional: o desafio da requalificação dos serviços. Katálysis, Florianópolis, SC. 2002. v.5, n.1, p.51-58. Disponível em $<$ https://periodicos.ufsc.br/index.php/katalysis/article/view/5870/5423>. Acesso em: 30 maio 2018.

NJAINE, K. et al. Redes de prevenção à violência: da utopia à ação. Ciência \& Saúde Coletiva, Rio de Janeiro, v.11, supl. 2006. Disponível

em:<http://www.scielo.br/scielo.php?script=sci_arttext\&pid=S1413-

$81232006000500020 \&$ Ing=en\&nrm=iso >. Acesso em: 14 maio de 2018.

https://doi.org/10.1590/S1413-81232006000500020

PINSKY, I.; BESSA, M. A. Adolescência e Drogas. São Paulo: Contexto, 2004.

RODRIGUES, K. O conceito de lugar: a aproximação da geografia com o indivíduo. In: ENCONTRO NACIONAL DA ASSOCIAÇÃO NACIONAL DE PÓS-GRADUAÇÃO E PESQUISA EM GEOGRAFIA, 9., 2015, Goiânia. Anais... Goiânia: ANPEGE, 2015. p.5036-5047. Disponível em:

<http://www.enanpege.ggf.br/2015/anais/arquivos/17/473.pdf>. Acesso em: 11 fev. 2018.

SANTOS, M. O lugar: encontrando o futuro. Revista de Urbanismo e Arquitetura. Salvador. 1996. v.4, n.1, p.34-39. Disponível em:

<http://www.portalseer.ufba.br/index.php/rua/article/view/3113/2230>. Acesso em: 20 jul. 2017.

SCHELL, K. et al. Photovoice as a Teaching Tool: Learning by Doing with Visual Methods.

International Journal of Teaching and Learning in Higher Education, Georgia, USA. v.21, p. 340352, 2009.

SILVA JUNIOR, A. G. Modelos tecnoassistenciais em saúde: o debate no campo da saúde coletiva. 2.ed. São Paulo: Hucitec, 2006.

SILVA, L. F. B.; CARVALHO, M. S. Praças como espaços para saúde: o caso da praça Nishinomiya (Londrina-Paraná). Hygeia, Uberlândia, MG. 2017. v.13, n.26, p.175-191. Disponível em: <http://www.seer.ufu.br/index.php/hygeia/article/view/39739>. Acesso em: 20 fev. 2018.

https://doi.org/10.14393/Hygeia132615 
O olhar dos adolescentes sobre a violência e o lazer nos territórios pelas lentes do photovoice
Ailton Souza Aragão Rosimár Alves Querino Luana Cristina Silveira Gomes Luciana Trajano da Silva Maria Carolina Bizinoto Caetano Otávio Loyola Martins Ana Angelina Amatângelo Oliveira Ana Carolina Graner Araújo Oliveira Maria Lopes Santos

Fabiano Henrique Oliveira Sabino

STRACK, R. W. et al. Developing a web-based tool using information and communication technologies to expand the reach and impact of photovoice. American Journal Health Education, Londres. 2015. v.46, n.4, p.192-195. Disponível em:

<https://www.ncbi.nlm.nih.gov/pmc/articles/PMC5026412/pdf/nihms747353.pdf> Acesso em: 14 ago. 2017. https://doi.org/10.1080/19325037.2015.1044585

STRACK, R. W.; MAGILL, C.; MCDONAGH K. Engaging youth through photovoice. Health Promotion Practice, San José, CA. 2004. v.5, n.1, p.49-58. Disponível em: <https://journals.sagepub.com/doi/pdf/10.1177/1524839903258015>. Acesso em: 19 mar. 2018. https://doi.org/10.1177/1524839903258015

VILLASANTE, T. R. Redes e alternativas: estratégias e estilos criativos na complexidade social. Rio de Janeiro: Vozes, 2002.

WANG, C. et al. Flint Photovoice: Community Building Among Youths, Adults, and Policymakers. American Journal of Public Health, 2004. v.94, n.1, p.911-913. Disponivel em: <https://www.ncbi.nlm.nih.gov/pmc/articles/PMC1448361/>. Acesso em: 04 jul. 2018. https://doi.org/10.2105/AJPH.94.6.911

WANG, C.; BURRIS, M. A. Photovoive: concept, methodology and use for participatory needs assessment. Health, Education \& Behavior, 1997. v.24, n.3, p.369-387. Disponivel em: <https://doi.org/10.1177/109019819702400309>. Acesso em: 14 jul. 2018. https://doi.org/10.1177/109019819702400309 